\title{
Complexity and State-transitions in Social Dependence Networks
}

\author{
GIULIANO PISTOLESI ${ }^{\mathrm{a}, \mathrm{b}, *}$ and PIERLUIGI MODESTI ${ }^{\mathrm{b}, \dagger}$

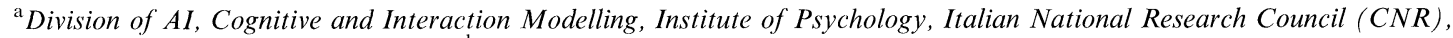 \\ V.le Marx 15-00137, Roma; ${ }^{\mathrm{b}}$ Division CEN/1, DCPT, Italian National Institute for Statistics (ISTAT), \\ Via Ravà 150-00142, Roma
}

(Received 6 June 2000)

\begin{abstract}
Computation of complexity in Social Dependence Networks is an interesting research domain to understand evolution processes and group exchange dynamics in natural and artificial intelligent Multi-Agent Systems. We perform an agent-based simulation by NET-PLEX (Conte and Pistolesi, 2000), a new software system able both to build interdependence networks tipically emerging in Multi-Agent System scenarios and to investigate complexity phenomena, i.e., unstability and state-transitions like Hopf bifurcation (Nowak and Lewenstein, 1994), and to describe social self-organization phenomena emerging in these artificial social systems by means of complexity measures similar to those introduced by Hubermann and Hogg (1986). By performing analysis of complexity in these kind of artificial societies we observed interesting phenomena in emerging organizations that suggest state-transitions induced by critical configurations of parameters describing the social system similar to those observed in many studies on state-transitions in bifurcation chaos (Schuster, 1988; Ruelle, 1989).
\end{abstract}

Keywords: Complexity; State-transitions phenomena; Social Dependence Networks; Multi-Agent Systems; Social simulation

\section{INTRODUCTION}

The paper takes a Multi-Agent System perspective on the issue of social complexity. In this paper, a theory of social dependence proposed elsewhere (Castelfranchi et al., 1992; Sichman et al., 1994; Conte et al., 1998; Conte and Pedone, 1998;
Pistolesi and Veneziano, 1998) is applied to simulate on computer the emergence of group dependence networks, and the formation of generalized forms of exchange. In this paper, the software system NET-PLEX is illustrated and applied to calculate multiagent dependence networks of variable complexity to investigate complexity

* Corresponding author. e-mail: pistoles@istat.it

${ }^{\dagger}$ e-mail: modesti@istat.it 
phenomena, i.e., unstability and state-transitions like Hopf bifurcation (Nowak and Lewenstein, 1994). This paper is a deeper investigation of our previous work with Dependence Networks complexity and NET-PLEX (Conte and Pistolesi, 2000).

\section{SOCIAL DEPENDENCE THEORY}

The work presented in this paper proceeds from the assumption that heterogeneous agents endowed with goals and actions and situated in a common world are involved in more or less complex and dynamic networks of relationships. In current agent systems, agents are often conceived of and designed as autonomous. However, they are not completely autonomous: agents may have goals which exceed or differ from their capacities to reach them. In particular, in teamwork, agents' autonomy is intrinsically limited. Analogously, in contexts like a market agents sell their resources in order to acquire those they are in need of.

Socially situated agents may depend on one another to achieve their own goals. In terms of the dependence theory, an agent $x$ is said to depend on some other agent $y$ with regard to one of its goal $p$ when (a) $x$ is not autonomous with regard to $p$ : it lacks at least one of the actions or resources necessary to achieve $p$, while (b) $y$ has the missing action/resource.

The theory of social dependence as presented in (Sichman et al., 1994) on the basis of a pre-existing model developed in (Castelfranchi et al., 1992) is summarized. Only the notion of dependence network, that is relevant for the present exposition, will be considered explicitly. For a complete formal expression, see (Skvoretz and Willer, 1993; Sichman et al., 1994; Conte et al., 1998).

The concept of dependence network is central in the dependence theory and it proceeds from that of agency consisting of a set of agents with their goals and actions. It is a data structure composed of the set of goals each agent wants to achieve, the set of actions she is able to perform. However, an agent may have a goal whose actions do not necessarily belong to her own set of actions, and therefore she may depend on others in order to carry on a certain plan, and achieve a certain goal. Therefore, agents are plunged into social networks, that is patterns of relationships holding among the goals and actions of a given set of agents. If an agent does not have all the actions to achieve a given goal, according to a set of plans, he may depend on the others for this goal. A dependence network represents in a single structure all of the social dependence relations within the agency. These networks can be used later to predict the emergence of group exchanges, and to evaluate the degree of complexity of the agency.

\section{COMPLEXITY IN SOCIAL DEPENDENCE NETWORKS}

In the present paper, a specific notion of social complexity is proposed, i.e., complexity in MultiAgent Systems, particularly with regards to Social Dependence Networks (Sichman et al., 1994; Conte et al., 1998).

\subsection{Structural Complexity}

Our research proceeded from the work of Hubermann and Hogg (1986) on complexity computation in morphological structures like graph trees and any other similar structure, like, in this case, a dependence network. They defined a recursive process to compute complexity of a tree considering all subtrees of the root and their diversity on isomorphical base. In the case of an artificial agent-based community, the complexity measures proposed by Hubermann and Hogg (1986) have to be adapted to fit the existence of dependence networks (i.e., similar to subtrees), which isomorphism needed to define their diversity can be stated as a function of both the number of agents belonging to a dependence network and the number of links inside that net. 
In particular, the concept of structural complexity of a social system we introduce in the theory of dependence networks (Conte and Pistolesi, 2000) is referred to as the number and dimension of dependence networks emerging in the Multi-Agent System. More precisely, given a social system $h_{i}$, its degree of interdependence complexity is said to vary as a positive function of the proportion between the number and size of the dependence networks emerging in it and the number of agents in $h_{i}$. In other words, it is hypothesized that the higher is the number of group dependence networks and the higher is the structural complexity of the social system. What is the relationship between this conceptualization of social complexity and the common intuition of complexity as a hindrance to the management of social systems, and therefore as a phenomenon to be reduced or controlled? This common intuition refers to functional or operational complexity, and it refers to the obstacles to the functioning of a system. In this paper, structural (possibly, organizational) complexity is indicated as a mean to address and manage the functional complexity, which investigation is one of the future objectives of this work. The emergence of dependence networks in a MultiAgent System, indeed, allows agents to find means to achieve their goals.

\subsection{Critical Parameters and State-transitions}

Among several sources, emergence of particular social phenomena depends on some parameters that are constant during evolution or whose values are controlled by factors that are external to the system but that determine to a great extent the character of the dynamics observed. Such parameters are often termed control parameters because they represent conditions or influences external to the system itself (Nowak and Lewenstein, 1994). With regards to Social Dependence Networks, the number of agents and the maximum number of goals/resources an agent possess are all control parameters critical for social organizations that should lead the system to change drastically organizational patterns emerging by self-organization. Since the number of agents in the social system is an endogenous control parameter, that is, a control parameter characterizing the uniqueness of the social system itself, we can consider the emergence of state-transictions phenomena as endogenous non-linear phenomena, that would emerge in a dynamical simulation too.

In nonlinear dynamics, smooth and small changes in control parameters may lead to dramatic qualitative changes in behavior (Nowak and Lewenstein, 1994). Therefore, the detection and analysis of radical changes in the patterns of behavior is critical to understand the influences of control parameters, and this important point is one of the basis of synergetics (Haken, 1978, 1982). Changes in patterns of behavior are called bifurcations. The theory of bifurcations is a very advanced and difficult branch of mathematics (Ruelle, 1989), and the observed bifurcation phenomena are many. However, analysis of correlation measures reckoned on the artificial social system evolution suggests the existence of an Hopf bifurcation phenomena, which characterizes changes in evolutionary trajectories from a stable point to a quasiperiodic cycle, i.e., a torus (Nowak and Lewenstein, 1994). A critical dimension of the social system activates the state-transition from a simple behavior in correlations among parameters of the system itself to an irregularly oscillating motion in correlations generated by superimposition of different waves or correlation behaviors. We underline that this behavior could be a road to chaos (i.e., a Landau-Hopf scenario as described in Nowak and Lewenstein, 1994): the process of bifurcation from a stable point to a torus attractor could continue, and more and more new frequencies could appear as tori bifurcate into tori in higher and higher dimensions, and the dynamics would become increasingly irregular. Such a scenario is highly probable when the phase space has a very large number of dimensions, and we think that this could be highly probable with the increasing of the number of agents, which determine the phase space in a social system. 
Given the system composed of:

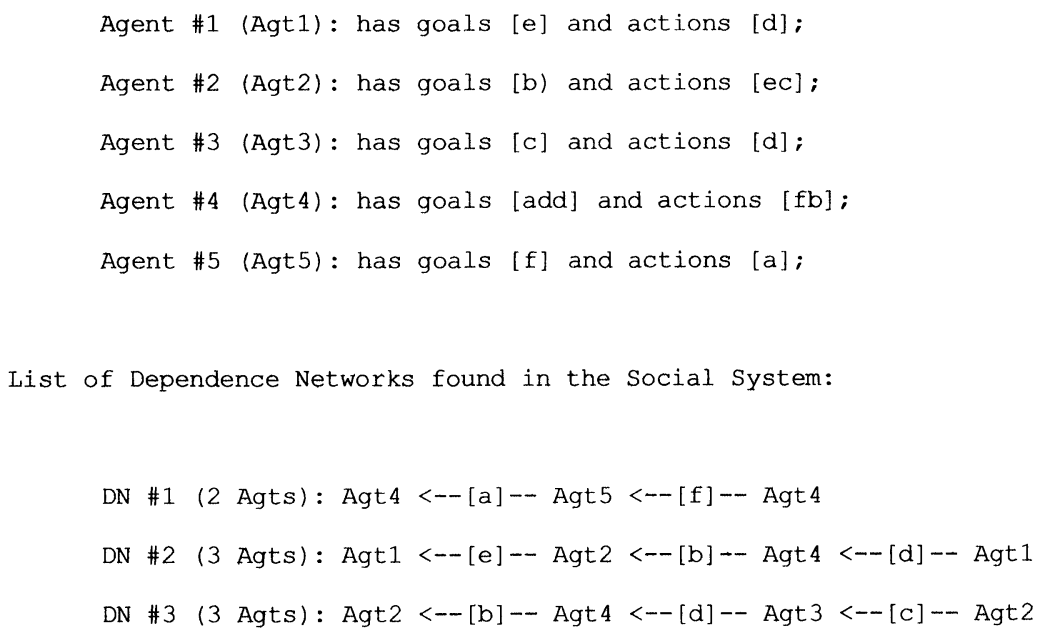

FIGURE 1 A sample of NET-PLEX output.

\subsection{Computing Complexity: The NET-PLEX Software Tool}

The NET-PLEX software tool (Conte and Pistolesi, 2000) is useful for:

- building artificial agent-based communities, i.e., networks of agents with goals and resources;

- calculating the dependence networks emerging from exchange links among those agents.

A typical running with NET-PLEX is composed of the following phases:

- Market generation: a finite number of agents is generated according to the desired dimension specified by the researcher; each agent is randomly assigned two lists, for its goals and actions respectively, according to a maximum limit specified by the researcher too; the agent's identity and the content of goals and actions are expressed by symbols (letters).

- Potential dependence links calculation: a list of partners upon which each agent depends is built.

- Dependence networks calculation: a smart backtracking algorithm applied to the lists of potential partners identifyes and extracts close networks of agents (dependence networks) giving rise to exchange groups; when an agent can chose the partner, its choice is made on heuristic basis.

- System's measures calculation: several individual (e.g., the Satisfaction Level) and social (e.g., Structural Complexity) measures are calculated.

A sample of the NET-PLEX output showing all dependence networks found is shown in Figure 1.

The structure of the artificial social system with dependence networks emerged is shown in Figure 2.

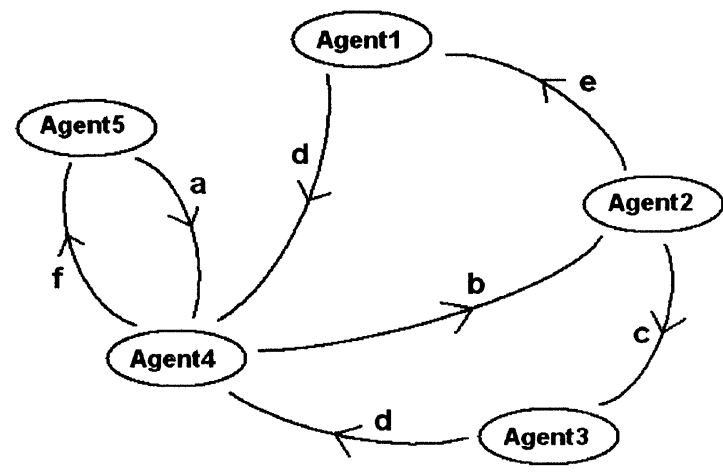

FIGURE 2 Social Dependence Networks emerged by selforganization. 


\subsection{Computing Complexity: Measures}

Simulations end after exchange execution, and a certain number of measures about the social system are calculated (see Fig. 3):

- Structural Complexity of the social system $(A)$ : indicates the number of links among agents effectively executed on the total number of agents, according to the following formula:

$$
S C=\frac{\sum l s \in D N s}{N_{\text {Agts }}}
$$

- where $l s$ are links between agents belonging to the set of dependence networks emerged (DNs) and $N_{\text {Agts }}$ is the total number of agents in the social system.

- The total number of group dependence networks emerged $(B)$.

- The average dimension of dependence networks emerged or average number of agent per dependence network $(C)$.

- The average Satisfaction Level in dependence networks: it is calculated according to the following formula:

$$
\bar{S}_{k}=\frac{\sum_{k} S_{k}}{M_{\text {Agts }} \in D N S}
$$

- where $M_{A g t s}$, in this case, is the total number of agents belonging to the set of dependence networks emerged ( $D N s)$, and $S_{k}$ is the Satisfaction Level of the $k$-agent in the system (see formula (5.4)).

- The average Satisfaction Level in the whole social system: it is calculated according to the following formula:

$$
\bar{S}_{k}=\frac{\sum_{k} S_{k}}{N_{\text {Agts }}}
$$

- where $N_{\text {Agts }}$ is the total number of agents in the social system, and $S_{k}$ is calculated (for the Satisfaction Level either in dependence networks and in the whole social system) by the following formula:

$$
S_{k}=\frac{l s_{k}}{g s_{k}}
$$

- where $l s_{k}$ is the number of links the $k$-agent forms with other agents in the social system, and $g_{s}$ is the number of goals the $k$-agent possesses.

According to the central hypothesis that dependence networks proved to be useful for both evaluating Multi-Agent structural complexity, and

\section{Correlation trends}

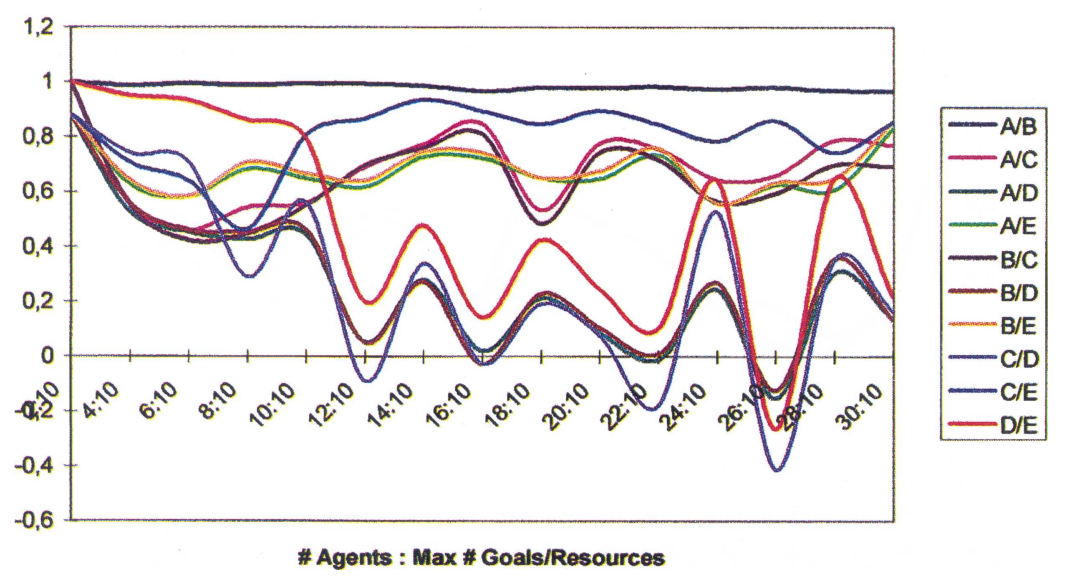

FIGURE 3 The dynamics of correlations along the different control parameter conditions. (See Color Plate II.) 
at the same time managing the operational complexity, we expected to find significant positive correlations among variable B-C-D, on one hand, and E-F, on the other.

\section{SIMULATIONS AND RESULTS}

The correlations among the variables described above, and the dynamics of these correlations under different conditions, have been checked. Experimental conditions varied with the combination of agents number, while maximum number of goals/actions per agent was fixed to 10. A fixed number of 30 experiments for each of the experimental conditions have been conducted, and the results are shown in Figure 3, which shows the dynamics of correlations.

Global significative correlations observed are:

- The correlation between the number of emerged dependence networks in the social system and their average dimension is 0.644: this result could seem somewhat surprising, because one should expect a negative correlation, that is, the higher is the number of dependence networks emerged, the smaller is the average dimension of networks themselves; however, we have to keep in mind that dependence networks can overlap each other, and therefore big networks (i.e., dependence networks with an high number of agents belonging to them) include a great number of smaller dependence networks, each time.

- The correlation between the structural complexity and the number of dependence networks emerged is 0.818 , while the correlation between the structural complexity and the average dimension of dependence networks is 0.610: this result is quite obvious, because structural complexity is calculated on the basis of those system measures.

- The correlation between the structural complexity and the average satisfaction level in the system is 0.637 : this is a very interesting result, because suggests that certain social organizations (i.e., certain configuration of dependence networks in the system) are more satisfying than others, like proved our previous studies (Pistolesi et al., 1997; Pistolesi and Veneziano, 1998).

- The correlation between the average dimension of dependence networks and the average satisfaction level in the system is 0.707: this result suggests that the greater are the dependence networks, the higher is the probability to include all agents belonging to the social system in a network, therefore improving the satisfaction level of the whole system.

Correlation trends



FIGURE 4 First group of correlations. (See Color Plate III.) 
On the basis of our previous study we did not consider the maximum number of goals/actions as a control parameter influencing the state-transitions of the social system, and we believe the number of agents that form the social system is the only control parameter critical for the emergence of those dynamics phenomena (Conte and Pistolesi, 2000).

About state-transitions phenomena in correlations dynamics, what is evident is a drastical change in patterns of behavior (e.g., an Hopf bifurcation) in the interval $[8,10]$ agents for almost all correlations, that we can consider a critical point for the state-transitions (our previous study suggested the critical point to be between the interval $[8,16]$ agents (Conte and Pistolesi, 2000). We can observe two main phase-opposed Hopf bifurcations in correlations: the former group of correlations include $\mathrm{A} / \mathrm{D}, \mathrm{C} / \mathrm{D}, \mathrm{B} / \mathrm{D}$ and $\mathrm{D} / \mathrm{E}$ (see Fig. 4), while the opposite one include $\mathrm{A} / \mathrm{C}, \mathrm{B} / \mathrm{C}$ (see Fig. 5) and A/E, B/E, C/E (see Fig. 6). Talking about the correlation between $\mathrm{D} / \mathrm{E}$ as a representative example of phenomena we observed, this

Correlation trends

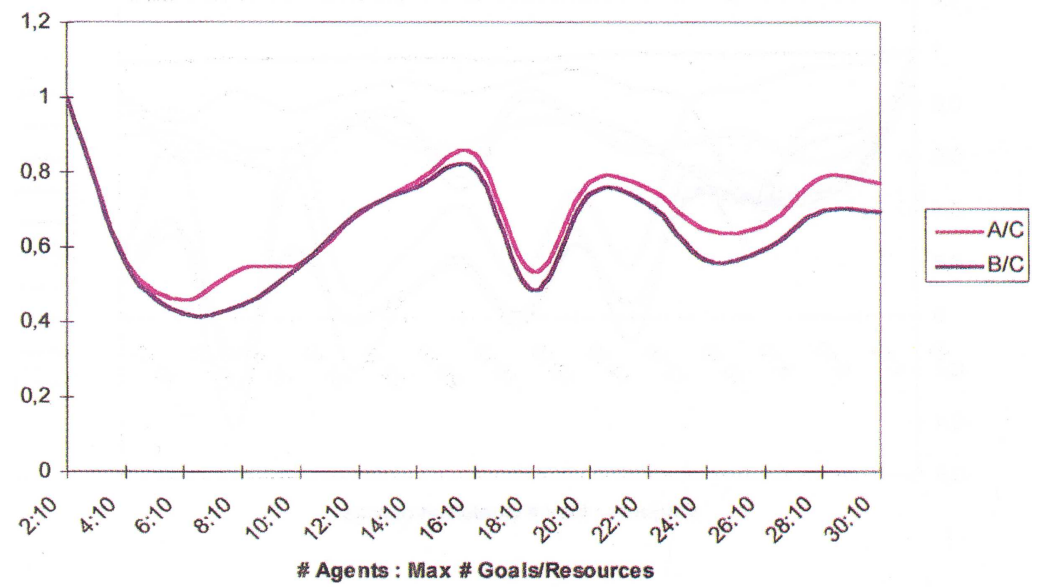

FIGURE 5 Second group of correlations. (See Color Plate IV.)

Correlation trends

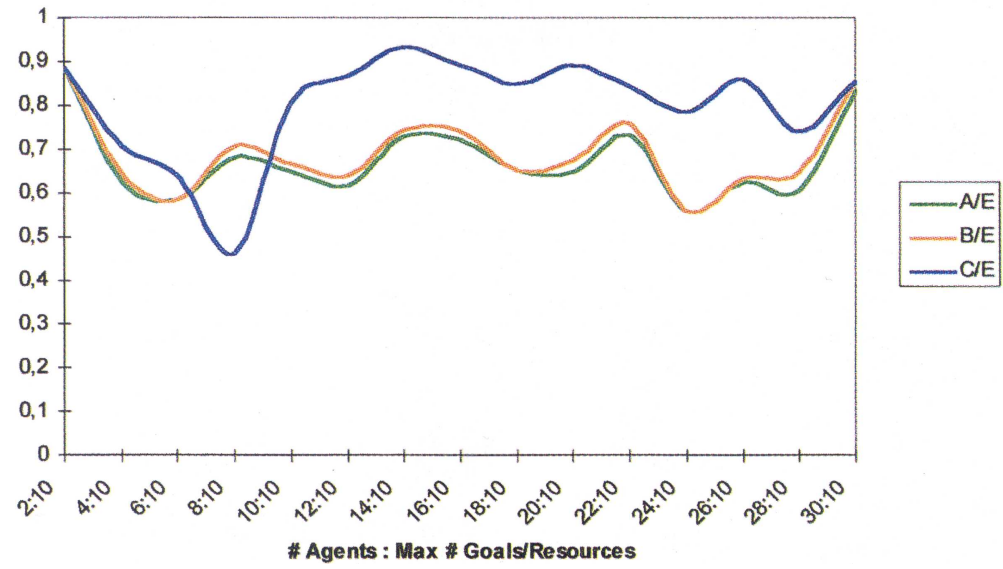

FIGURE 6 Third group of correlations. (See Color Plate V.) 
suggests us that there exists a critical equilibrium among emerged dependence networks and the social system dimension. This equilibrium is clearly critical in determining the social organizations emerged from evolution, particularly for a dimension of the social system higher than 10 agents, and the dynamics could continue to bifurcate into multiple tori with high probability, like hypotesized in the Landau-Hopf route to chaos (Nowak and Lewenstein, 1994).

\section{CONCLUSIONS}

The approach presented in this paper may have several advantages for Multi-Agent coordination and cooperation, particularly by helping to predict the emergence of partnerships in dynamic networks of heterogeneous exchange agents.

Moreover, some unstability phenomena were investigated and suggested us that the number of agents in a social system, i.e., the dimension of the system, is the main control parameter inducing state-transitions (i.e., Hopf bifurcations) and possibly route to chaos (through a Landau-Hopf scenario) in patterns of behavior of the system itself. Emergence of these phenomena take place when dependence networks organizations connect heterogeneous agents in a social system, that is, when are present networks of agents which depend each other to achieve their goals (i.e., one of the motivational roots of cooperative behavior in Multi-Agent Systems). These phenomena are interesting in order to understand the critical equilibrium in the self-organization process of a social system, given particular control parameter conditions, but more study will be necessary, particularly to verify the route to chaos hypothesis with the increasing of the dimension of the social system over 30 agents.

\section{References}

Castelfranchi, C., Cesta, A. and Miceli, M. (1992) Dependence Relations in Multi-Agent Systems. In: Decentralized AI Vol. 3 (Demazeau, Y. and Werner, E. Eds.), Elsevier, NorthHolland.

Conte, R. and Pistolesi, G. (in press) NET-PLEX: An AgentBased Simulator of Dependence Networks Complexity and Group Exchange. In: Proceedings of the 16th IMACS World Congress 2000 on Scientific Computation, Applied Mathematics and Simulation EPFL. Lousanne, Switzerland, $21-$ 25 August, 2000 (http://imacs2000.epfl.ch).

Conte, R. and e Pedone, R. (1998) Finding the best partner: The PART-NET system. In: Multi-Agent Systems and Social Simulation (Sichman, J. S., Conte, R. and Gilbert, N. G. Eds.). Berlin, Springer.

Conte, R., Veneziano, V. and Castelfranchi, C. (1998) The Computer Simulation of Partnership Formation. Computational and Mathematical Organization Theory, 4, $293-315$.

Haken, H. (Ed.) (1982) Order and Chaos in Physics, Chemistry, and Biology. Springer-Verlag, Berlin.

Haken, H. (1978) Synergetics. Springer-Verlag, Berlin.

Hubermann, B. A. and Hogg, T. (1986) Complexity and Adaptation. Physica 22D, pp. 376-384.

Nowak, A. and Lewenstein, M. (1994) Dynamical Systems: A Tool for Social Psychology? In: Dynamical Systems in Social Psychology (Vallacher, R. R. and Nowak, A. Eds.). Academic Press, San Diego (CA.), pp. 17-53.

Pistolesi, G. and Veneziano, V. (1998) A Computational Investigation in the Cognitive Microstructure of Social Systems: The Role of Rationality Models in the Emergence of Partnerships. Cybernetica, 41, 217-234.

Pistolesi, G., Veneziano, V. and Castelfranchi, C. (1997) Global vs. Local Social Search in the Formation of Coalitions. In: Simulating Social Phenomena (Conte, R., Hegselmann, R. and Terna, P. Eds.), Springer-Verlag, Berlin, pp. 203-209.

Ruelle, D. (1989) Elements of Differentiable Dynamics and Bifurcation Theory. Academic Press, New York.

Schuster, H. G. (1988) Deterministic Chaos: an Introduction. $\mathrm{VCH}$, Weinheim.

Sichman, J. S., Conte, R., Castelfranchi, C. and Demazeau, Y. (1994) A social Reasoning Mechanism Based on Dependence Networks. In: Proceedings of the 11th European Conference on Artificial Intelligence (Cohn, A. G. Ed.). Wiley \& Sons, Baffins Lane, England, pp. 188-192.

Skvoretz, J. and Willer, D. (1993) Exclusion and power: a test of four theories of power in exchange networks. American Sociological Review, 58, 801-818. 


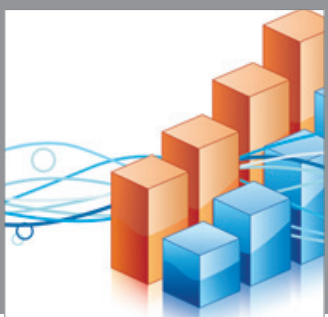

Advances in

Operations Research



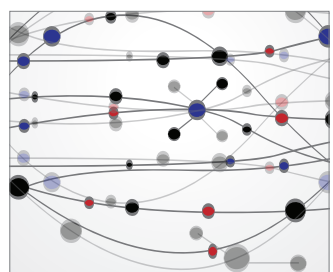

\section{The Scientific} World Journal


International Journal of

Mathematics and

Mathematical

Sciences
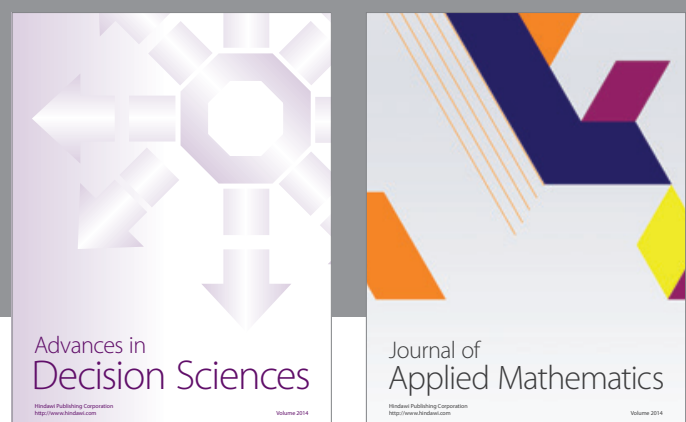

Journal of

Applied Mathematics
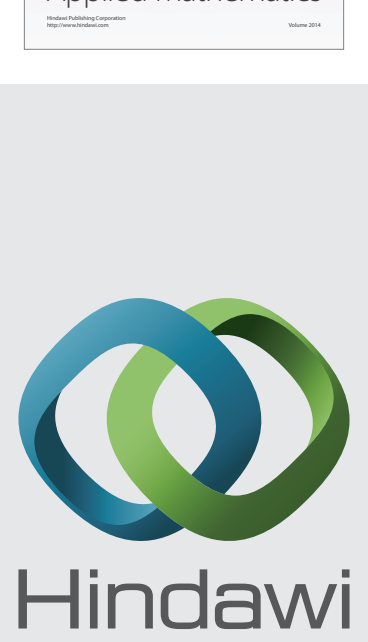

Submit your manuscripts at http://www.hindawi.com
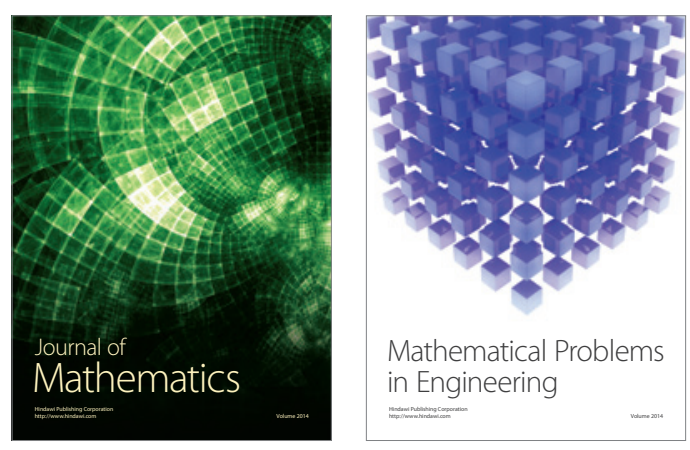

Mathematical Problems in Engineering
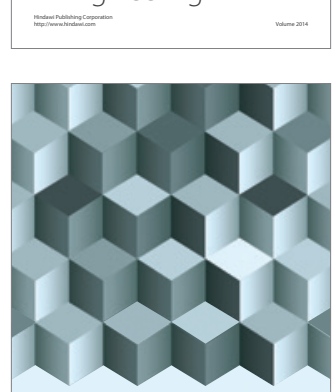

Journal of

Function Spaces


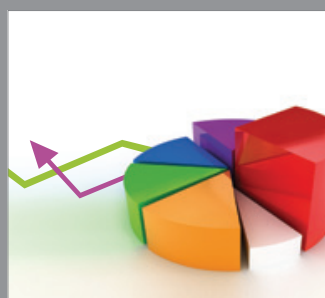

ournal of

Probability and Statistics

Promensencen
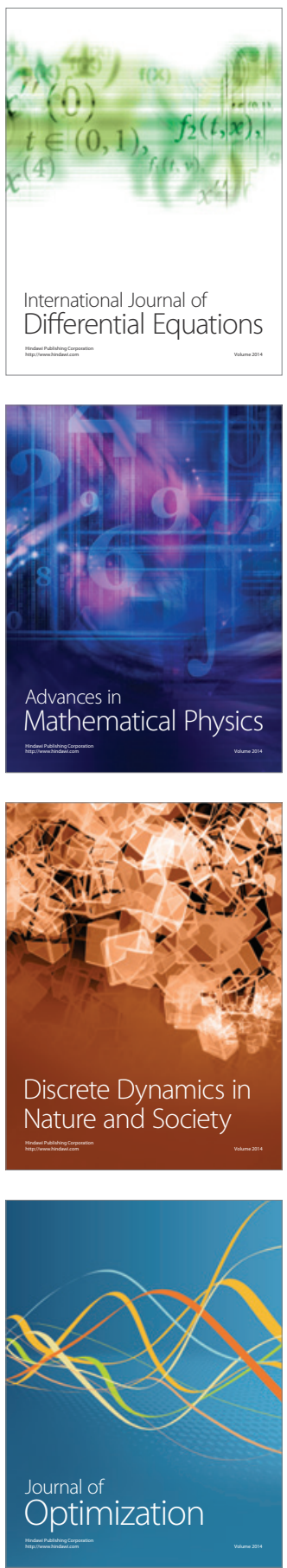